Z Epileptol 2011 $\cdot 24: 245-246$

DOI 10.1007/s10309-011-0204-8

Online publiziert: 19. Oktober 2011

(c) Springer-Verlag 2011

\author{
S. Noachtar ${ }^{1}$ M. Hoppe ${ }^{2}$ \\ ${ }^{1}$ Epilepsie-Zentrum, Neurologische Klinik und Poliklinik, Klinikum \\ der Universität München-Großhadern, München \\ ${ }^{2}$ Abt. für EEG-und Video-Diagnostik, Epilepsie-Zentrum Bethel, \\ Epilepsiekliniken Krankenhaus Mara gGmbH, Bielefeld
}

\title{
Elektroenzephalographie bei Epilepsie
}

\section{Alte Rebe und neuer Wein}

Das EEG spielt in der Diagnostik der Epilepsien unverändert eine große Rolle. In vielen anderen Bereichen der Neurologie ist dies nicht mehr der Fall, da neuere Methoden wie Dopplersonographie und Magnetresonanztomographie wesentlich sensitiver und spezifischer sind, z. B. für die Diagnostik von Durchblutungsstörungen oder Hirntumoren. Vor allem die epilepsiechirurgische Diagnostik hat die Entwicklung des EEG in den letzten Jahrzehnten vorangetrieben. Der technische Fortschritt der digitalen Signalverarbeitung hat geholfen, viele Beschränkungen der Anfangsjahre zu überwinden. Inzwischen sind digitale EEG-Geräte Standard, und es wurden ausgefeilte Registrierungs- sowie Analyseverfahren entwickelt. Vieles davon erlaubt den breiten Einsatz des EEG im klinischen Alltag. Nach wie vor kommt dem OberflächenEEG in seinen verschiedenen Variationen (Routine-EEG, Langzeitableitungen, EEG-Video-Monitoring) eine Schlüsselstellung in der Diagnostik anfallsartiger Ereignisse zu. Seine diagnostische Wertigkeit hängt von einer hohen Aufzeichnungsqualität und kompetenter Interpretation ab. Es ist unter Epileptologen unbestritten, dass Fehler bei der Epilepsiediagnostik nicht selten auf Unsicherheiten und Fehlern bei der EEG-Analyse beruhen. Dies wird durch manche unscharfe Grenzen in der Beurteilung des EEG begünstigt. Man kann beim EEG nicht wie z. B. bei den evozierten Potenzialen mit Standardabweichungen gesunde von pa- thologischen Befunden abgrenzen. Hier sind viel Erfahrung und Abwägung komplexer Zusammenhänge erforderlich. Die Interobserver-Übereinstimmung ist daher beim EEG nicht so gut, wie wir uns das wünschen würden. Die ersten beiden Themen dieses Sonderhefts sind deswegen als propädeutische Beiträge zu verstehen und der klassischen visuellen EEGAnalyse, -Befunderstellung und -Interpretation gewidmet. Sie sind als Hilfe zu verstehen, alle Möglichkeiten des EEG im klinischen Alltag systematisch auszuschöpfen, aber auch seine Grenzen zu erkennen und Fallgruben bei der EEG-Analyse zu vermeiden. Zwei weitere Beiträge stellen methodische Erweiterungen der diagnostischen Möglichkeiten des EEG mithilfe der Quellenlokalisation und Identifizierung neuer Muster in höheren Frequenzbereichen vor. Quellenlokalisation beruht auf einer präziseren Potenzialfeldanalyse mithilfe geeigneter Algorithmen und kann die visuelle Analyse bei manchen Fragestellungen sinnvoll ergänzen. Die Hochfrequenzoszillationen liegen weit über den im Oberflächen-EEG zugänglichen Frequenzen (obere Grenzfrequenz $70 \mathrm{~Hz}$ ). Ihnen scheint Bedeutung in der Identifizierung der epileptogenen Zone zuzukommen; ihr Stellenwert muss jedoch zukünftig noch weiter evaluiert werden.

Der fünfte Beitrag umfasst noch einen anderen klinischen Bereich, in dem das EEG bis heute diagnostisch wichtig ist: die Diagnostik von Patienten mit Bewusst- seinsstörungen bzw. Enzephalopathien. Insbesondere in der Diagnostik des nonkonvulsiven Status epilepticus und seiner Abgrenzung zu Enzephalopathien oder psychogenen Störungen ist das EEG nach wie vor durch nichts zu ersetzen.

Wir hoffen, mit diesem Themenheft EEG bei Epilepsie die Bedeutung einer altbewährten und immer noch unverzichtbaren Untersuchungsmethode mit ihren Stärken und Schwächen zu verdeutlichen. Es würde uns freuen, wenn es gelänge, bei der Leserschaft Interesse am EEG (wieder) zu erwecken und - zum Wohle unserer Patienten - zur kritischen Auseinandersetzung mit dieser Untersuchungsmethode beizutragen.

\section{Korrespondenzadresse \\ Prof. Dr. S. Noachtar}

Epilepsie-Zentrum, Neurologische Klinik und

Poliklinik, Klinikum der Universität

München-Großhadern

Marchioninistr. 15, 81377 München

noa@med.uni-muenchen.de

\section{Dr. M. Hoppe}

Abt. für EEG-und Video-Diagnostik, Epilepsie-Zentrum Bethel, Epilepsiekliniken Krankenhaus Mara gGmbH

Maraweg 21, 33617 Bielefeld

Matthias.Hoppe@mara.de

Interessenkonflikt. Der korrespondierende Autor gibt an, dass kein Interessenkonflikt besteht. 


\section{Hier steht eine Anzeige.}

亚 Springer 